\title{
Informe Horizon 2015: de la expansión del aprendizaje híbrido a la cultura de la innovación en las IES
}

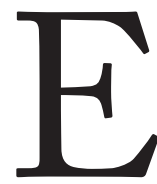
$\mathrm{n}$ la era digital y en el contexto educativo vigente, los ambientes de aprendizaje mediados por las Tecnologías de la Información y Comunicación, cobran cada día más fuerza, como lo revela el Informe Horizon 2015 Educación Superior, que desde el 2004

40 publica The New Media Con2015 sortium ahora en conjunto con EDUCAUSE Learning Initiative, estudio que ubica el incremento del uso del aprendizaje mixto o híbrido como una tendencia que se posiciona en la educación superior a corto plazo, es decir 1-2 años "a partir de las mejores prácticas en los métodos en línea y presenciales, el aprendizaje híbrido (blended learnig) está en aumento en las universidades y escuelas".

El informe señala que actualmente hay una mayor comprensión de las potencialidades del aprendizaje híbrido, resaltando su flexibilidad y la posibilidad de acceso e integración de recursos multimedia, el uso de herramientas para comunicación e interacción síncronas y asíncronas así como la incorporación con soltura de otras tecnologías de punta.

Ello conlleva a que más universidades se interesen en la generación y desarrollo de cursos en línea para reemplazar o completar los cursos convencionales, cien por ciento ofrecidos bajo metodologías presenciales.

El documento refiere que de acuerdo a la United State's National Center for Education Statistic uno de cada 10 estudiantes estaba inscrito en cursos en línea; asimismo, los estudios del Babson Research Group indican que 7.1 millones de estudiantes de Estados Unidos participan en cursos en línea (NMC, 2015, p.16).

El tema calidad de la educación en línea es de alta prioridad, por ello el informe también refleja los resultados de investigaciones al respecto "los investigadores identificaron la claridad, la autenticidad, la unidad, el suspenso, la profundidad, la economía, la proporción, la viveza, el brillo, la sensibilidad, el énfasis, la auto- ridad, el flujo y precisión como los parámetros de referencia definitivos" (NMC, 2015, p.16) . En ese sentido, un tema prioritario para las universidades es el mejoramiento del diseño y ejecución de los cursos en línea que ofrecen.

En el ámbito de los profesores que se enfrentan hoy día al reto de combinar docencia presencial y docencia en línea, este reporte enfatiza en tareas que deben promover y llevar a cabo los buenos profesores que se desempeñen en escenarios formativos híbridos "estimular las actividades sociales y el pensamiento crítico dentro del entorno en línea" (NMC, 2015, p.16). De igual forma, dada la diversidad de estudiantes, deben utilizar formas diversas para el aprendizaje de sus alumnos.

Nuevos espacios y metodologías para el aprendizaje De la mano de esa primer tendencia que es evidente en instituciones nacionales como la Universidad Nacional Autónoma de Honduras, UNAH, donde hace más de cinco año se orientan esfuerzos a nivel 
normativo y de proyectos innovadores para el desarrollo de procesos formativos con el componente presencial y virtual o en línea, evidenciado en su oferta académica virtual de dos licenciaturas y un técnico universitario y la opción de cursar asignaturas en línea como parte de los programas en modalidad presencial (CTUNAH, 2008, UNAH, 2014; DIE, 2014); se posiciona también a corto plazo el rediseño de los espacios de aprendizaje.

Es una realidad inobjetable que la educación superior de este siglo es testigo de una oleada de modelos educativos y pedagógicos emergentes, relacionados con el cambio de un paradigma que ha dejado atrás la educación bancaria -que tanto cuestionó el educador brasileño Paulo Freire- y el rol protagónico del profesor como el mayor detentor de conocimiento, hacia un nuevo enfoque paradigmático que centra su quehacer en el aprendizaje y que confiere al docente un rol de facilitador, guía, tutor y diseñador de estrategias y de recursos didácticos para el aprendizaje de sus alumnos. Por su lado los alumnos asumen protagonismo activo y responsable para su aprendizaje.

$\mathrm{Al}$ respecto el Informe del NMC 2015 precisa que la formación universitaria se ve irrumpida por modelos como: el de aula invertida que da lugar a un aprendizaje más activo; metodologías didácticas, como el aprendizaje basado en proyectos con un alto grado de flexibilidad, movilidad y utilización de diversos dispositivos tecnológicos y móviles.
El aula de clases tradicional de corte físico con un pizarrón en frente y las sillas colocadas una detrás de otra, parecen ir en proceso de desaparición, dando el paso a las "salas inteligentes” y diseño de espacios abiertos y flexibles, que propician el trabajo colaborativo y potencian la ubicuidad, convirtiendo el espacio donde se encuentre el alumno en un ambiente propicio para aprender muy cerca de su realidad social, económica y cultural, lo que vuelve más real y significativo el aprendizaje.

Desde esta óptica muchos de los espacios informarles del campus universitario pueden ser habilitados para convertirse en espacios para el aprendizaje "donde los estudiantes se congregan y pueden trabajar de forma más productiva". Estos espacios se habilitan con muebles cómodos, tomacorrientes para cargar móviles y pantallas LCD para la conexión de ordenadores portátiles.

A medida que la educación superior continúe alejándose de la programación basada en la clase tradicional y hacia más situaciones prácticas, las aulas universitarias comenzarán a parecerse a los ambientes sociales y de trabajo del mundo real que facilitan interacciones y la resolución de problemas interdisciplinarios (NMC, 2015, p.p. 18).

En esta nueva reconfiguración de los espacios para el aprendizaje la típica cátedra o podio se traslada del sitial preferencial delantero hacia el centro, los tradicionales pupitres se sustituyen por sillas móviles que permite a los estudiantes trabajar colaborativamente con otros compañeros y con otros grupos "cada mesa puede estar tecnológicamente habilitada con pizarras interactivas u otras superficies de marcado".

\section{Tendencias con plazo de adopción de 3 y 5 años El Informe Horizon 2015} precisa además tendencias a mediano y largo y plazo, es decir a tres y cinco años, las que también muestran evidencias de su impacto en la educación superior. A mediano plazo se sitúan el crecimiento del enfoque sobre la medición del aprendizaje y la expansión de los recursos abiertos. A largo plazo el avance en la cultura del cambio y la innovación y el incremento de la colaboración interinstitucional.

Como posibilitadora permanentemente de la pertinencia y la mejora continua, es de resaltar el posicionamiento a cinco años de la cultura para la innovación que puntualiza el Informe Horizon 2015, al señalar que las instituciones de educación superior deben adoptar estructuras que faciliten la flexibilidad, estimulen la creatividad y el pensamiento empresarial. Apuntan que actualmente las empresas están innovando para convertirse siempre en necesarias para sus consumidores, y al igual que éstas las universidades deben considerar ese comportamiento que ya observa resultados exitosos en este sector económico "en el negocio de la educación superior, los consumidores son los estudiantes y existe una necesidad de aten- 
derlos mejor, al tiempo que sus expectativas y comportamiento van evolucionando" (NMC, 2015, p.8).

En esta línea de pensamiento, el desarrollo tecnológico, la investigación y la innovación debe ser potenciadas $y$ valoradas en las universidades, asimismo se debe favorecer la participación activa de profesores y estudiantes en este campo que tiene amplios espacios de posicionamiento a nivel mundial.

La visión de futuro debe impregnar el liderazgo de las instituciones de educación superior, según esta tendencia de largo plazo "hace falta una clase de liderazgo particularmente visionario para construir entornos que estén equipados que puedan cambiar rápidamente los procesos y estrategias como lo hacen las startups. Si estos modelos organizacionales están bien diseñados, las universidades pueden experimentar una ejecución más eficiente de nuevas prácticas y pedagogías (NMC, 2015, p.9).

El reporte del NMC también destaca las tecnologías que ocuparán un lugar importante como herramientas para el aprendizaje en las universidades. Se colocan a corto plazo el uso de los diversos dispositivos móviles como el celular y las tabletas; a mediano plazo los makerspaces y a cinco años el Internet de las cosas. Es importante remarcar que estos desarrollos tecnológicos van de la mano con estrategias pedagógicas innovadoras que posibilitan poner en el centro
Cuadro No. 1. Tendencias a corto y largo plazo en la educación superior según Informe Horizon 2015 del NMC

\begin{tabular}{|c|c|}
\hline Plazo de adopción & $\begin{array}{c}\text { Tendencia } \\
\text { Corto plazo } \\
\text { (1-2 años) }\end{array}$ \\
$\begin{array}{c}\text { Medio plazo } \\
\text { (3-4 años) }\end{array}$ & $\begin{array}{c}\text { Incremento del uso del aprendizaje mixto o hibrido. } \\
\text { Crecimiento del enfoque sobre la medición del } \\
\text { aprendizaje. }\end{array}$ \\
\hline $\begin{array}{c}\text { Largo plazo } \\
\text { (5+años) }\end{array}$ & \begin{tabular}{c} 
Expansión de la Recursos Educativos Abiertos. \\
\hline
\end{tabular} \\
\hline
\end{tabular}

Fuente: NMC Informe Horizon 2015

Cuadro No. 2. Desarrollos Tecnológicos 2015-2020

\begin{tabular}{|c|c|}
\hline Plazo de adopción & Desarrollos en la tecnología \\
\hline $\begin{array}{c}\text { Corto plazo } \\
(1 \text { año o menos })\end{array}$ & Trae tu propio dispositivo (BYOD) \\
\hline Medio plazo & El aula invertida \\
\hline$\left(2^{-} 3\right.$ años $)$ & Makerspaces \\
\hline $\begin{array}{c}\text { Largo plazo } \\
(4-5 \text { años })\end{array}$ & Tecnología "Wearable" \\
\hline & Tecnología de aprendizaje adaptativo \\
\hline
\end{tabular}

Fuente: NMC Informe Horizon 2015

Cuadro No. 3. Seis desafíos para las universidades

\begin{tabular}{|c|c|}
\hline Nivel & Desafíos \\
\hline \multirow{2}{*}{ Solucionables } & Mezcla del aprendizaje formal e informal \\
\cline { 2 - 2 } & Mejora de la alfabetización digital \\
\hline \multirow{2}{*}{ Difíciles } & Personalización del aprendizaje \\
\cline { 2 - 2 } & Enseñanza del pensamiento complejo \\
\hline \multirow{2}{*}{ Muy difíciles } & Modelos de educación en competencia \\
\cline { 2 - 2 } & Premiar la enseñanza \\
\hline
\end{tabular}

Fuente: NMC Informe Horizon 2015

de la formación universitaria el aprendizaje y el desarrollo de competencias pertinentes a las exigencias laborales y cultura digital del presente siglo.

Un aspecto altamente significativo de los reportes e informes que por más de una década viene generando NMC (20042015), son los desafíos que en el marco de esas tendencias y desarrollos tecnológicos, a su juicio deben enfrentas las instituciones de educación superior, ubicándolo en tres niveles solucionables, difíciles y muy difíciles. En el cuadro No. 3 se detallan esos desafíos.

En base a la realidad educativa de diversos países, como los de la región centroamericana, quizás exceptuando a Costa Rica, es importante enfatizar desafíos presentes como la mejora de la alfabetización digital, particularmente a nivel de los profesores y estudiantes, para aprovechar todas las posibilidades del mundo tecnológico y poder desenvolverse con soltura en la dinámica de una era digital. 
Ello conlleva tener clara comprensión que no basta con saber teclear una computadora, o enviar mensajes vía correo electrónico, la alfabetización para una era digital, conlleva otras implicaciones y desarrollo de competencia informacionales. Encaminarse a este desafío solucionable implica un compromiso con la pertinencia profesional, social y cultural de directivos, docentes, estudiantes y todos los empleados de las universidades que deben dar el salto hacia una universidad pertinente a los tiempos actuales.

$Y$ finalmente parece necesario una reflexión final sobre el desafío de las universidades para visibilizar o recompensar en mayor medida la enseñanza, como lo sostiene el Informe Horizon 2015, en tanto ha sido la investigación la que ha ocupado el lugar de preeminencia "es un sentir generalizado en el mundo académico que las credenciales de investigación son un activo más valioso que el talento y la habilidad como docente" (pág. 32).

En ese sentido, este estudio considera el poco valor que las universidades prestan a la enseñanza y por ende a la docencia de calidad, genera un ambiente poco favorable para desarrollar y poner en marcha pedagogías y estrategias didáctica efectivas.

Los profesores adjuntos y estudiantes sienten la peor parte de este desafío, ya que los contratos de enseñanza pura son subestimados $y$ mal pagados, y los alumnos están sujetos a los estilos de enseñanza anticuados de los investigadores principales de la universidad (NMC, 2015, pág.32).

Sobre este debate entre docencia e investigación el documento del NMC refiere un informe del año 2013 de la Comisión Europea sobre la Mejora de la Calidad de la Enseñanza y Aprendizaje en las Instituciones de Educación Superior, que expone tres puntos sobre este reto: la necesidad de priorizar la enseñanza por encima de la investigación, la formación de los profesores para que enseñen con estándares de primer nivel y la necesidad de que los legisladores y líderes intelectuales "impulsen a las instituciones de educación superior a revisar sus objetivos para hacer de la enseñanza una piedra angular".

De acuerdo a este informe hay ejemplos que muestra cómo este desafío está siendo atendido. Se menciona el caso del gobierno de Australia que asignó recursos financieros específicos para la Promoción de la Excelencia en la Enseñanza y el Aprendizaje de la Educación Superior 2014-2015.

En Canadá donde la carga de trabajo entre la docencia y la investigación es más equilibrada, según este reporte -para ejemplo- administradores de la Universidad de York consideran contratar a 200 profesores que estarán centrados en la enseñanza "los administradores han justificado la necesidad de crear más puestos centrados en la enseñanza sobre todo en las universidades públicas regionales antes que en las instituciones de inves- tigación más emblemáticas" (NMC, 2015, pág.37).

Otro elemento que se señala es que una enseñanza de calidad también potencia las competencias para la investigación a través del usos de pedagogías más eficaces, citando el caso de la Universidad de Texas donde los profesores del Departamento de Matemáticas han utilizado la estrategia del aprendizaje basado en la indagación con el propósito que los alumnos se conviertan en generadores activos de conceptos matemáticos.

\section{Referencias}

Instituto Nacional de Tecnologías Educativas y de Formación del Profesorado, INTEF(2015). Resumen Informe Horizon 2015 Educación Superior. Recuperado de: http://blog.educalab.es/intef/wp-content/ uploads/sites/4/2015/08/ Resumen_Horizon_Universidad_2015_INTEF_ agosto_2015.pdf

Johnson, L., Adams Becker, S., Estrada, V. and Freeman, A. (2015). NMC Horizon Report: Educación Superior 2015, Austin, Texas: The New Media Consortiun. Recuperado de: http://www. puce.edu.ec/documentos/ pucevirtual/2015-Horizon-Report.pdf

UNAH (2014). Normas Académicas. Tegucigalpa, Honduras

UNAH (2008). Acuerdo No. 348. Comisión de Transición. Tegucigalpa, Honduras.

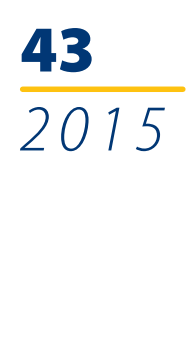

